\title{
Non-Markovian Quantum Trajectories Versus Master Equations: Finite Temperature Heat Bath
}

\author{
Ting $\mathrm{Yu}^{*} \mathrm{t}$ \\ Rochester Theory Center for Optical Science and Engineering \\ and \\ Department of Physics and Astronomy, \\ University of Rochester, \\ Rochester, NY 14627, USA
}

(Dated: 29 March 2004)

\begin{abstract}
The interrelationship between the non-Markovian stochastic Schrödinger equations and the corresponding non-Markovian master equations is investigated in the finite temperature regimes. We show that the general finite temperature non-Markovian trajectories can be used to derive the corresponding non-Markovian master equations. A simple, yet important solvable example is the well-known damped harmonic oscillator model in which a harmonic oscillator is coupled to a finite temperature reservoir in the rotating wave approximation. The exact convolutionless master equation for the damped harmonic oscillator is obtained by averaging the quantum trajectories relying upon no assumption of coupling strength or time scale. The master equation derived in this way automatically preserves the positivity, Hermiticity and unity.

PACS numbers: 03.65.Yz, 42.50.Lc, 05.40.Jc
\end{abstract}

Keywords: Open quantum systems; Non-Markovian quantum trajectories, Master equations

\footnotetext{
* Also at Department of Physics, Queen Mary College, University of London, London, E1 4NS, UK

$\dagger$ Electronic address: ting@pas.rochester.edu
} 


\section{INTRODUCTION}

Open systems are generic models for the study of quantum dynamics in the sense that a real quantum system is either difficult to be isolated from the influence of its environment or is deliberately put in touch with some purposely engineered devices in order to make a measurement [1, 2]. In any case, the system and the environment, initially independent, will become entangled due to the interaction, then the system state will not remain in a pure state and the dynamics is described by a non-unitary process. In many physically relevant cases, a typical open quantum system normally involves a small system of interest coupled to a large system with large number of degrees of freedom, commonly known as heat bath, reservoir or generally environment. Traditionally, the dynamics of a system of interest is described by a Lindblad master equation which can often be derived if the standard BornMarkov approximation is assumed [2, 3, 4, 5]. Moreover, it turns out that such a Lindblad master equation also allows to be unravelled into various stochastic Schrödinger equations known as quantum trajectories [6, 7, 8, 9, 10, 11, 12]. Both the master equations of the Lindblad form and their stochastic unravelings have become an integral part of theories of open quantum systems. However, when the heat bath memory effects are relevant, such as in the cases of high-Q cavity, atom laser or structured environment, where the Born-Markov approximation ceases to be valid, hence the dynamics of the open quantum system must be described by a non-Markovian process [13, 14]. It is long known that the derivation of a non-Markovian master equation is a formidable task [15, 16].

Recent research on open quantum systems has suggested that, alternatively, the nonMarkovian dynamics may be described by a diffusive stochastic Schrödinger equation known as non-Markovian quantum trajectories or quantum state diffusion equation 17, 18, 19, 20, 21, 22, 23, 24, 25, 26, 27, 28, 29]. For an $N$-dimensional Hilbert space, the stochastic Schrödinger equation evolves an $N$-dimensional vector, so offers numerical advantages over a master equation which evolves an $N \times N$ density matrix. Very recently, we have shown that, beyond the numerical advantages and the conceptual merits, the non-Markovian stochastic Shrödinger equations may also provide a powerful tool for deriving the corresponding nonMarkovian master equations. This idea has been explored in several distinct cases including a two-level atom interacting with a zero-temperature heat bath [20, 28], and a Brownian particle coupled linearly to a finite temperature heat bath via the position variable commonly 
known as quantum Brownian motion model [21, 29, 30]. The purpose of this paper is to extend this research to more general finite temperature regimes where the Lindblad operator is not a Hermitian operator. In this paper, we take the damped harmonic oscillator as our primary example. This simple model is of great interest in quantum optics because it is an essential ingredient in the theoretical investigations of various quantum optical experiments. We show here in detail that the non-Markovian quantum trajectories allow the derivation of the exact convolutionless master equation irrespective of the coupling strength, the separated time scales, or the special distributions of the environmental frequencies.

The organization of the paper is as follows. In Sec. 피, we introduce both zero and finite temperature stochastic Schrödinger equations. In Sec. III, we show how to derive an exact master equation from the finite temperature quantum trajectories. In Sec. IV. we establish the stochastic Schrödinger equation for the damped harmonic oscillator and show that the corresponding exact convolutionless master equation can be obtained by averaging the solutions to the non-Markovian stochastic Schrödinger equation without any approximations, in particular, without Markov approximation. We conclude this paper in Sec. D

\section{NON-MARKOVIAN STOCHASTIC SCHRÖDINGER EQUATION}

\section{A. Zero-temperature $(T=0)$}

Our model consists of a system of interest coupled linearly to a large number of harmonic oscillators with distributed eigenfrequencies $\omega_{\lambda}$ and creation and annihilation operators $b_{\lambda}^{\dagger}, b_{\lambda}$. The quantum Hamiltonian for the system plus reservoir can be typically written as (we set $\hbar=1$ )

$$
\begin{aligned}
H_{\mathrm{tot}} & =H_{\mathrm{sys}}+H_{\mathrm{int}}+H_{\mathrm{bath}} \\
& =H_{\mathrm{sys}}+\sum_{\lambda}\left(g_{\lambda}^{*} L^{\dagger} b_{\lambda}+g_{\lambda} L b_{\lambda}^{\dagger}\right)+\sum_{\lambda} \omega_{\lambda} b_{\lambda}^{\dagger} b_{\lambda},
\end{aligned}
$$

where $g_{\lambda}$ are the coupling constants and the system operator $L$ coupled to the environment is often called the Lindblad operator.

For the open quantum system described by (1), the linear non-Markovian quantum state diffusion (QSD) equation has been derived from the formal solution of Schrödinger equation 
for the total system in a special representation [18]:

$$
i \partial_{t}\left|\Psi_{t}\right\rangle=H_{\text {tot }}(t)\left|\Psi_{t}\right\rangle
$$

where $\left|\Psi_{t}\right\rangle$ stands for the pure state vector for the total system and $H_{\text {tot }}(t)$ is the Hamiltonian $H_{\text {tot }}$ in the interaction representation with respect to the free bath Hamiltonian:

$$
\begin{aligned}
H_{\mathrm{tot}}(t) & =e^{i H_{\mathrm{bath}} t}\left(H_{\mathrm{sys}}+\sum_{\lambda}\left(g_{\lambda}^{*} L^{\dagger} a_{\lambda}+g_{\lambda} L a_{\lambda}^{\dagger}\right)\right) e^{-i H_{\mathrm{bath}} t} \\
& =H_{\mathrm{sys}}+\sum_{\lambda}\left(g_{\lambda}^{*} L^{\dagger} a_{\lambda} e^{-i \omega_{\lambda} t}+g_{\lambda} L a_{\lambda}^{\dagger} e^{i \omega_{\lambda} t}\right) .
\end{aligned}
$$

In this subsection, let us assume that the initial pure state of the system and the environment is taken to be

$$
\left|\Psi_{0}\right\rangle=\left|\psi_{0}\right\rangle \otimes\left|0_{1}\right\rangle \otimes\left|0_{2}\right\rangle \cdots \otimes\left|0_{\lambda}\right\rangle \otimes \cdots
$$

with an arbitrary system state $\left|\psi_{0}\right\rangle$ and the environment in the ground state $|0\rangle$. By using a Bargmann coherent state basis for the environmental degrees of freedom: $\left|z_{\lambda}\right\rangle=$ $\exp \left\{z_{\lambda} a_{\lambda}^{\dagger}\right\}\left|0_{\lambda}\right\rangle$ and the resolution of the identity

$$
I_{\lambda}=\int \frac{d^{2} z_{\lambda}}{\pi} \mathrm{e}^{-\left|z_{\lambda}\right|^{2}}\left|z_{\lambda}\right\rangle\left\langle z_{\lambda}\right|
$$

the total state $\left|\Psi_{t}\right\rangle$ can be expressed as

$$
\left|\Psi_{t}\right\rangle=\int \frac{d^{2} z}{\pi} \mathrm{e}^{-|z|^{2}}\left|\psi_{t}\left(z^{*}\right)\right\rangle \otimes|z\rangle
$$

where $\left.|z\rangle=\left|z_{1}\right\rangle \otimes z_{2}\right\rangle \otimes \cdots \otimes\left|z_{\lambda}\right\rangle \cdots, d^{2} z=d^{2} z_{1} d^{2} z_{2} \cdots d^{2} z_{\lambda} \cdots$ and $|z|^{2}=\sum_{\lambda}\left|z_{\lambda}\right|^{2}$. Then the resultant pure state for the system of interest $\left|\psi_{t}\left(z^{*}\right)\right\rangle$ was shown to satisfy the following equation [17, 18]:

$$
\partial_{t} \psi_{t}=-i H_{\mathrm{sys}} \psi_{t}+L z_{t}^{*} \psi_{t}-L^{\dagger} \int_{0}^{t} d s \alpha(t-s) \frac{\delta \psi_{t}}{\delta z_{s}^{*}} .
$$

where $\alpha(t-s)=\sum_{\lambda}\left|g_{\lambda}\right|^{2} e^{-i \omega_{\lambda}(t-s)}$ is the bath correlation function, and $z_{t}^{*}=-i \sum_{\lambda} g_{\lambda}^{*} z_{\lambda}^{*} e^{i \omega_{\lambda} t}$ is a colored, complex Gaussian noise with $\mathcal{M}\left[z_{t}\right]=\mathcal{M}\left[z_{t} z_{s}\right]=0$ and $\mathcal{M}\left[z_{t}^{*} z_{s}\right]=\alpha(t-s)$. Note here $\mathcal{M}[\cdot]$ is the statistical mean over the Gaussian process $z_{t}$. By construction,

$$
\rho_{t}=\mathcal{M}\left[\left|\psi_{t}\right\rangle\left\langle\psi_{t}\right|\right]=\int \frac{d^{2} z}{\pi} e^{-|z|^{2}}\left|\psi_{t}\right\rangle\left\langle\psi_{t}\right|
$$

The linear non-Markovian QSD equation (7) is valid for a zero-temperature heat bath, and also for a finite temperature heat bath with the condition $L=L^{\dagger}$. In general, if $L \neq L^{\dagger}$, we shall see below that the finite temperature non-Markovian QSD equation takes a more complicated form. 


\section{B. Finite temperature $(T \neq 0)$}

The finite temperature stochastic Schrödinger equation or non-Markovian QSD equation can be obtained by mapping the system with the total Hamiltonian (II) and an initial thermal state to an extended system with a vacuum state such that the zero temperature stochastic Schrödinger equation for the extended system is equivalent to the situation where the heat bath is at finite temperature [18, 31]. To be specific, for the total Hamiltonian (11), we assume that the heat bath is in a thermal equilibrium state at temperature $T$, with density operator

$$
\rho_{\text {bath }}(0)=\frac{e^{-\beta H_{\text {bath }}}}{Z},
$$

where $Z=\operatorname{Tr}\left[e^{-\beta H_{\text {bath }}}\right]$ is the partition function and $\beta=1 / k_{B} T$.

Now we introduce a fictitious heat bath with the bosonic operators $c_{\lambda}, c_{\lambda}^{\dagger}$, which has no direct interaction with the system, ensuring that after tracing over the fictitious variables, the initial state of the original bath will be in the thermal state $\rho_{\text {bath }}(0)$. The total Hamiltonian with two independent heat baths is given by

$$
\mathcal{H}_{\text {tot }}=H_{\text {sys }}+\sum_{\lambda}\left(g_{\lambda}^{*} L^{\dagger} b_{\lambda}+g_{\lambda} L b_{\lambda}^{\dagger}\right)+\sum_{\lambda} \omega_{\lambda} b_{\lambda}^{\dagger} b_{\lambda}-\sum_{\lambda} \omega_{\lambda} c_{\lambda}^{\dagger} c_{\lambda} .
$$

The boson Bogoliubov transformation formally couples the system of interest to two set of bosonic operators $d_{\lambda}, d_{\lambda}^{\dagger}$ and $e_{\lambda}, e_{\lambda}^{\dagger}$ :

$$
\begin{aligned}
& b_{\lambda}=\sqrt{\bar{n}_{\lambda}+1} d_{\lambda}+\sqrt{\bar{n}_{\lambda}} e_{\lambda}^{\dagger}, \\
& c_{\lambda}=\sqrt{\bar{n}_{\lambda}+1} e_{\lambda}+\sqrt{\bar{n}_{\lambda}} d_{\lambda}^{\dagger},
\end{aligned}
$$

where $\bar{n}_{\lambda}$ is the mean thermal occupation number of quanta in mode $\omega_{\lambda}$ :

$$
\bar{n}_{\lambda}=\frac{1}{\exp \left(\hbar \omega_{\lambda} / k_{B} T\right)-1} .
$$

The transformed Hamiltonian $\mathcal{H}_{\text {tot }}^{\prime}$ in terms of $d_{\lambda}, e_{\lambda}$ is then given by

$$
\begin{aligned}
\mathcal{H}_{\text {tot }}^{\prime}= & H_{\text {sys }}+\sum_{\lambda} \sqrt{\bar{n}_{\lambda}+1}\left(g_{\lambda}^{*} L^{\dagger} d_{\lambda}+g_{\lambda} L d_{\lambda}^{\dagger}\right)+\sum_{\lambda} \omega_{\lambda} d_{\lambda}^{\dagger} d_{\lambda} \\
& +\sum_{\lambda} \sqrt{\bar{n}_{\lambda}}\left(g_{\lambda}^{*} L^{\dagger} e_{\lambda}^{\dagger}+g_{\lambda} L e_{\lambda}\right)-\sum_{\lambda} \omega_{\lambda} e_{\lambda}^{\dagger} e_{\lambda} .
\end{aligned}
$$

Thus we have mapped the finite temperature problem into a zero temperature one with the initial vacuum state denoted by $|0\rangle=|0\rangle_{d} \otimes|0\rangle_{e}$, satisfying $d_{\lambda}|0\rangle=0, e_{\lambda}|0\rangle=0$. 
With the new Hamiltonian (14), the finite temperature problem has been reduced to a zero temperature one. Thus the resultant pure state for the system of interest $\psi_{t}=\left|\psi_{t}\left(z^{*}, w^{*}\right)\right\rangle$ satisfies the following stochastic Schrödinger equation with two independent noises $z_{t}^{*}, w_{t}^{*}$ :

$$
\begin{aligned}
\partial_{t} \psi_{t}= & -i H_{\mathrm{sys}} \psi_{t}+L z_{t}^{*} \psi_{t}-L^{\dagger} \int_{0}^{t} d s \alpha_{1}(t-s) \frac{\delta \psi_{t}}{\delta z_{s}^{*}} \\
& +L^{\dagger} w_{t}^{*} \psi_{t}-L \int_{0}^{t} d s \alpha_{2}(t-s) \frac{\delta \psi_{t}}{\delta w_{s}^{*}}
\end{aligned}
$$

where

$$
\begin{aligned}
& \alpha_{1}(t-s)=\sum_{\lambda}\left(\bar{n}_{\lambda}+1\right)\left|g_{\lambda}\right|^{2} e^{-i \omega_{\lambda}(t-s)}, \\
& \alpha_{2}(t-s)=\sum_{\lambda} \bar{n}_{\lambda}\left|g_{\lambda}\right|^{2} e^{i \omega_{\lambda}(t-s)}
\end{aligned}
$$

are the bath correlation functions and

$$
\begin{aligned}
& z_{t}^{*}=-i \sum_{\lambda} \sqrt{\bar{n}_{\lambda}+1} g_{\lambda}^{*} z_{\lambda}^{*} e^{i \omega_{\lambda} t}, \\
& w_{t}^{*}=-i \sum_{\lambda} \sqrt{\bar{n}_{\lambda}} g_{\lambda}^{*} w_{\lambda}^{*} e^{-i \omega_{\lambda} t}
\end{aligned}
$$

are two independent, colored, complex Gaussian noises satisfying

$$
\begin{aligned}
& \mathcal{M}\left[z_{t}\right]=\mathcal{M}\left[z_{t} z_{s}\right]=0, \mathcal{M}\left[z_{t}^{*} z_{s}\right]=\alpha_{1}(t-s), \\
& \mathcal{M}\left[w_{t}\right]=\mathcal{M}\left[w_{t} w_{s}\right]=0, \mathcal{M}\left[w_{t}^{*} w_{s}\right]=\alpha_{2}(t-s)
\end{aligned}
$$

Note here $\mathcal{M}[\cdot]$ is the statistical mean over the Gaussian processes $z_{t}^{*}$ and $w_{t}^{*}$. Again, by construction, $\rho_{t}=\mathcal{M}\left[\left|\psi_{t}\right\rangle\left\langle\psi_{t}\right|\right]$. In the zero temperature limit $T \rightarrow 0$, we have $\alpha_{1}(t-$ $s) \rightarrow \sum_{\lambda}\left|g_{\lambda}\right|^{2} \exp \left(-i \omega_{\lambda}(t-s)\right)$ and $\alpha_{2}(t-s) \rightarrow 0$, then equation (15) reduces to the simple zero-temperature case (17). However, in this paper, without explicit statement, we will always work in the finite temperature regimes. From equation (15), we see that the finite temperature heat bath has induced both the spontaneous transitions and stimulated transitions, moreover, it also gives rise to an absorptive process caused by taking thermal quanta from the heat bath.

The stochastic Schrödinger equation (15) can be greatly simplified by using the ansatz,

$$
\begin{aligned}
\frac{\delta \psi_{t}}{\delta z_{s}^{*}} & =O_{1}\left(t, s, z^{*}, w^{*}\right) \psi_{t} \\
\frac{\delta \psi_{t}}{\delta w_{s}^{*}} & =O_{2}\left(t, s, z^{*}, w^{*}\right) \psi_{t}
\end{aligned}
$$


Then equation (15) takes a more compact form:

$$
\begin{aligned}
\partial_{t} \psi_{t}= & -i H_{\mathrm{sys}} \psi_{t}+L z_{t}^{*} \psi_{t}-L^{\dagger} \bar{O}_{1}\left(t, z^{*}, w^{*}\right) \psi_{t} \\
& +L^{\dagger} w_{t}^{*} \psi_{t}-L \bar{O}_{2}\left(t, z^{*}, w^{*}\right) \psi_{t}
\end{aligned}
$$

where $\bar{O}_{i}(i=1,2)$ denote

$$
\bar{O}_{i}\left(t, z^{*}, w^{*}\right)=\int_{0}^{t} \alpha_{i}(t-s) O_{i}\left(t, s, z^{*}, w^{*}\right) d s, \quad(i=1,2) .
$$

We can determine the operators $O_{1,2}\left(t, s, z^{*}, w^{*}\right)$ in (22) and (23) from the following 'consistency conditions',

$$
\partial_{t} \frac{\delta \psi_{t}}{\delta z_{s}^{*}}=\frac{\delta}{\delta z_{s}^{*}} \partial_{t} \psi_{t}, \quad \partial_{t} \frac{\delta \psi_{t}}{\delta w_{s}^{*}}=\frac{\delta}{\delta w_{s}^{*}} \partial_{t} \psi_{t},
$$

together with the initial conditions:

$$
O_{1}\left(t=s, s, w^{*}, z^{*}\right)=L, \quad O_{2}\left(t=s, s, w^{*}, z^{*}\right)=L^{\dagger}
$$

From the consistency conditions, we may get the evolution equations for $O_{1}=$ $O_{1}\left(t, s, z^{*}, w^{*}\right)$ and $O_{2}=O_{2}\left(t, s, z^{*}, w^{*}\right)$

$$
\begin{aligned}
\partial_{t} O_{1}= & {\left[-i H_{\mathrm{sys}}+L z_{t}^{*}+L^{\dagger} w_{t}^{*}-L^{\dagger} \bar{O}_{1}-L \bar{O}_{2}, O_{1}\right] } \\
& -L^{\dagger} \frac{\delta \bar{O}_{1}}{\delta z_{s}^{*}}-L \frac{\delta \bar{O}_{2}}{\delta z_{s}^{*}}
\end{aligned}
$$

and

$$
\begin{aligned}
\partial_{t} O_{2}= & {\left[-i H_{\mathrm{sys}}+L z_{t}^{*}+L^{\dagger} w_{t}^{*}-L^{\dagger} \bar{O}_{1}-L \bar{O}_{2}, O_{2}\right] } \\
& -L^{\dagger} \frac{\delta \bar{O}_{1}}{\delta w_{s}^{*}}-L \frac{\delta \bar{O}_{2}}{\delta w_{s}^{*}} .
\end{aligned}
$$

It should be remarked here that the O-operators can be determined in many interesting situations [18, 29], and moreover, that the approximate O-operators can always be obtained by invoking a perturbation technique [20, 21, 28]

\section{NON-MARKOVIAN MASTER EQUATION AT FINITE TEMPERATURE}

From the non-Markovian QSD equation (24), one may take the statistical mean to derive the master equation, and gets

$$
\begin{aligned}
\partial_{t} \rho_{t}= & -i\left[H_{\mathrm{sys}}, \rho_{t}\right]+\left[L, \mathcal{M}\left\{P_{t} \bar{O}_{1}^{\dagger}\left(t, z^{*}, w^{*}\right)\right\}\right]-\left[L^{\dagger}, \mathcal{M}\left\{\bar{O}_{1}\left(t, z^{*}, w^{*}\right) P_{t}\right\}\right] \\
& +\left[L^{\dagger}, \mathcal{M}\left\{P_{t} \bar{O}_{2}^{\dagger}\left(t, z^{*}, w^{*}\right)\right\}\right]-\left[L, \mathcal{M}\left\{\bar{O}_{2}\left(t, z^{*}, w^{*}\right) P_{t}\right\}\right]
\end{aligned}
$$


This is the general master equation at finite temperature. Although this last result is still not a closed evolution equation for $\rho_{t}$, it nevertheless shows how a convolutionless master equation may result from our knowledge of the operators $O_{i}\left(t, s, z^{*}, w^{*}\right),(i=1,2)$.

As we mentioned before, in the case of finite temperature with $L \neq L^{\dagger}$, the O-operators will generally contain noises $z_{t}^{*}, w_{t}^{*}$. However, we begin with some special cases in which the operators $O_{i}\left(t, s, z^{*}, w^{*}\right)$ turn out to be independent of the noises $z_{t}^{*}, w_{t}^{*}$. In such a case, we may write $\bar{O}_{i}\left(t, z^{*}, w^{*}\right)=\bar{O}_{i}(t)$ and (30) is indeed a convolutionless, closed master equation

$$
\partial_{t} \rho_{t}=-i\left[H_{\mathrm{sys}}, \rho_{t}\right]+\left[L, \rho_{t} \bar{O}_{1}^{\dagger}(t)\right]+\left[\bar{O}_{1}(t) \rho_{t}, L^{\dagger}\right]+\left[L^{\dagger}, \rho_{t} \bar{O}_{2}^{\dagger}(t)\right]+\left[\bar{O}_{2}(t) \rho_{t}, L\right]
$$

for the non-Markovian open system.

As a direct application of (31), let's discuss an important dephasing process: the system Hamiltonian commutes with the Lindblad operator $\left[H_{\text {sys }}, L\right]=0$. More generally, we may consider a solvable model with $\left[L, H_{\mathrm{sys}}\right]=i \kappa I, L=L^{\dagger}, \kappa=$ real constant, and $I$ is the identity operator. For this case, it is easy to check that the solutions to (28) and (29) are given by

$$
\begin{aligned}
& O_{1}\left(t, s, z^{*}, w^{*}\right)=L-\kappa(t-s), \\
& O_{2}\left(t, s, z^{*}, w^{*}\right)=L-\kappa(t-s) .
\end{aligned}
$$

By simply inserting (32) and (33) into (31), a closed convolutionless master equation is immediately obtained:

$$
\frac{d}{d t} \rho_{t}=-i\left[H_{\mathrm{sys}}, \rho_{t}\right]+f(t)\left[L \rho_{t}, L\right]+f^{*}(t)\left[L, \rho_{t} L\right]+g(t)\left[\rho_{t}, L\right]+g^{*}(t)\left[L, \rho_{t}\right],
$$

where the time-dependent coefficients are given by

$$
f(t)=\int_{0}^{t} \alpha(t-s) d s \text { and } g(t)=\kappa \int_{0}^{t} \alpha(t-s)(t-s) d s
$$

Note here that the finite temperature correlation function $\alpha(t-s)$ is given by

$$
\alpha(t-s)=\alpha_{1}(t-s)+\alpha_{2}(t-s)=\eta(t-s)+i \nu(t-s)
$$

where

$$
\begin{aligned}
& \eta(t-s)=\sum_{\lambda}\left|g_{\lambda}\right|^{2} \operatorname{coth}\left(\frac{\omega_{\lambda}}{2 k_{B} T}\right) \cos \left[\omega_{\lambda}(t-s)\right] \\
& \nu(t-s)=-\sum_{\lambda}\left|g_{\lambda}\right|^{2} \sin \left[\omega_{\lambda}(t-s)\right] .
\end{aligned}
$$


The master equation (34) can be immediately applied to qubits decoherence and disentanglement [32]. To be specific, let's consider the dephasing process in a quantum register containing $N$ non-interacting qubits. It is known that one of the most important decoherence processes in the quantum registers is that $N$ qubits collectively interact with some environmental noise which only randomly interrupts the phase of each qubit. This problem can be effectively modeled by choosing $H_{\text {sys }}=\sum_{i=1}^{N} \omega_{i} \sigma_{i}^{z}$ and $L=\sum_{i=1}^{N} \sigma_{i}^{z}$ in the general Hamiltonian (11), where $\sigma_{i}^{z}$ denotes the Pauli matrix for the $i$ th qubit. The master equation for such a dephasing model is given by (34) with $\kappa=0$.

Of particular interest is the Markov approximation where $\alpha_{1}(t-s)=\gamma_{1} \delta(t-s), \alpha_{2}(t-s)=$ $\gamma_{2} \delta(t-s)$, then $\bar{O}_{1}=\frac{\gamma_{1}}{2} L, \bar{O}_{2}=\frac{\gamma_{2}}{2} L^{\dagger}$, so the resulting master equation takes the well known Lindblad form

$$
\begin{aligned}
\partial_{t} \rho_{t}=-i\left[H_{\mathrm{sys}}, \rho_{t}\right] & +\frac{\gamma_{1}}{2}\left(2 L \rho_{t} L^{\dagger}-L^{\dagger} L \rho_{t}-\rho_{t} L^{\dagger} L\right) \\
& +\frac{\gamma_{2}}{2}\left(2 L^{\dagger} \rho_{t} L-L L^{\dagger} \rho_{t}-\rho_{t} L L^{\dagger}\right) .
\end{aligned}
$$

Before ending this section we have two remarks in order. First, let's note that the nonMarkovian property of a convolutionless master equation (31) or more generally (30) is characterized by the time-dependent coefficients. Second, if the O-operators appearing in (22) and (23) or in (30) do contain noises, as seen from the next section, things become much more complicated. There is no general guideline how to derive the closed convolutionless master equation from (30). However, it has been shown that the evolution equations for the O-operators $O_{i}\left(t, s, z^{*}, w^{*}\right)$ with respect to $s$ rather than $t$ are essential for such a derivation. Here we believe that "The existence of a set of uncoupled evolution equations for the Ooperators with respect to $s$ " is a very powerful ansatz. Obviously, this ansatz remains to be tested in more examples [24, 29].

\section{THE DAMPED HARMONIC OSCILLATOR}

\section{A. Stochastic Schrödinger equation for the damped harmonic oscillator}

In this section we show that the non-Markovian quantum QSD approach is versatile enough to handle the general finite temperature cases with $L \neq L^{\dagger}$. To simplify the calculations as much as possible, we consider here the simplest model of this kind but still 
worth studying - the damped harmonic oscillator model. The model consists of a harmonic oscillator with frequency $\Omega$, coupled linearly to a large number of harmonic oscillators: $H_{\mathrm{sys}}=\Omega a^{\dagger} a, L=a$. We assume that the heat bath is at finite temperature $T$. This model has been discussed in various contexts (see, e.g., [3, 6] ). In what follows, we use this model to show explicitly how the finite temperature master equation can be derived directly from the non-Markovian quantum trajectory equation. For the damped harmonic oscillator at zero temperature, it has been shown that $w_{t}^{*}=0$ and $\bar{O}_{1}=F(t) a, \bar{O}_{2}=0$ [18, 29]. Thus the zero-temperature non-Markovian master equation is immediately obtained from (31). For the finite temperature heat bath, as to be seen below, the O-operators $O_{1}$ and $O_{2}$ for the damped harmonic oscillator will contain noises $z_{t}^{*}$ and $w_{t}^{*}$, hence things become much more involved. We emphasize that, for a quantum system with $L \neq L^{\dagger}$, the dependence of the O-operators on the noises is a generic feature for the finite temperature cases. First, note that the exact quantum state diffusion equation for the damped harmonic oscillator can be written as

$$
\begin{aligned}
\partial_{t} \psi_{t}= & -i \Omega a^{\dagger} a \psi_{t}+a z_{t}^{*} \psi_{t}-a^{\dagger} \bar{O}_{1}\left(t, z^{*}, w^{*}\right) \psi_{t} \\
& +a^{\dagger} w_{t}^{*} \psi_{t}-a \bar{O}_{2}\left(t, z^{*}, w^{*}\right) \psi_{t}
\end{aligned}
$$

where $\bar{O}_{i}$ are defined in (25) .

Crucial to the solution of QSD equation (40) is the explicit determination of the Ooperators. It is easy to check that the following ansatz will give rise to the solutions of equation (28) and (29),

$$
\begin{aligned}
& O_{1}\left(t, s, z^{*}, w^{*}\right)=f_{1}(t, s) a+\int_{0}^{t} d s^{\prime} j_{1}\left(t, s, s^{\prime}\right) w_{s^{\prime}}^{*} \\
& O_{2}\left(t, s, z^{*}, w^{*}\right)=f_{2}(t, s) a^{\dagger}+\int_{0}^{t} d s^{\prime} j_{2}\left(t, s, s^{\prime}\right) z_{s^{\prime}}^{*}
\end{aligned}
$$


where $f_{i}(t, s)$ and $j_{i}\left(t, s, s^{\prime}\right)$ satisfy the coupled nonlinear integro-differential equations:

$$
\begin{gathered}
\partial_{t} f_{1}(t, s)-i \Omega f_{1}(t, s)-f_{1}(t, s) \int_{0}^{t} d s^{\prime} \alpha_{1}\left(t-s^{\prime}\right) f_{1}\left(t, s^{\prime}\right) \\
-f_{1}(t, s) \int_{0}^{t} d s^{\prime} \alpha_{2}\left(t-s^{\prime}\right) f_{2}\left(t, s^{\prime}\right)+\int_{0}^{t} d s^{\prime} \alpha_{2}\left(t^{\prime}-s\right) j_{2}\left(t, s^{\prime}, s\right)=0 \\
\partial_{t} f_{2}(t, s)+i \Omega f_{2}(t, s)+f_{2}(t, s) \int_{0}^{t} d s^{\prime} \alpha_{1}\left(t-s^{\prime}\right) f_{1}\left(t, s^{\prime}\right) \\
+f_{2}(t, s) \int_{0}^{t} d s^{\prime} \alpha_{2}\left(t-s^{\prime}\right) f_{2}\left(t, s^{\prime}\right)+\int_{0}^{t} d s^{\prime \prime} \alpha_{1}\left(t-s^{\prime \prime}\right) j_{1}\left(t, s^{\prime \prime}, s\right)=0 \\
\partial_{t} j_{1}\left(t, s, s^{\prime}\right)-f_{1}(t, s) \int_{0}^{t} d s^{\prime \prime} \alpha_{1}\left(t-s^{\prime \prime}\right) j_{1}\left(t, s^{\prime \prime}, s^{\prime}\right)=0 \\
\partial_{t} j_{2}\left(t, s, s^{\prime}\right)+f_{2}(t, s) \int_{0}^{t} d s^{\prime \prime} \alpha_{2}\left(t-s^{\prime \prime}\right) j_{2}\left(t, s^{\prime \prime}, s^{\prime}\right)=0
\end{gathered}
$$

with the initial values:

$$
\begin{aligned}
& f_{1}(t=s, s)=1, \quad f_{2}(t=s, s)=1, \\
& j_{1}\left(t=s, s, s^{\prime}\right)=0, \quad j_{2}\left(t=s, s, s^{\prime}\right)=0, \\
& j_{1}(t, s, t)=-f_{1}(t, s), \quad j_{2}(t, s, t)=f_{2}(t, s) .
\end{aligned}
$$

Indeed we have seen from (41) and (42) that, in the case of finite temperature, the Ooperators generally involve the noises, even though at zero-temperature they do not.

With the above evolution equations for $f_{i}(t, s)$ and $j_{i}\left(t, s, s^{\prime}\right)$, we are able to simulate the system dynamics by solving the non-Markovian QSD equation (40) 34]. But our aim in this paper is to take the quantum trajectories as theoretical tools rather than as numerical applications. Clearly, the solutions of the above nonlinear coupled equations are hard to deal with. In the next subsection, we will show remarkably that the evolution equations for $f_{i}(t, s)$ and $h_{i}\left(t, s, s^{\prime}\right)$ with respect to $s$ can be turned into a set of first-order linear uncoupled equations.

\section{B. Decoupling evolution equations for O-operators}

The functions appearing in equations (43)-(46), as they stand, are very difficult to handle analytically. However, as shown in [29] (also see 24]), great simplification may arise through investigating the dependence of the functions on $s$ rather than $t$. It is proved here that the evolution equations for the O-operators with respect to $s$ form an uncoupled set of linear 
differential equations. The key observation comes from the Heisenberg operator approach to the non-Markovian QSD [24, 29]. For our purpose in this paper, the main task here is to find the evolution equations for

$$
A(s)=\langle z|\left\langle w\left|\mathcal{U}_{t} a(s)\right| 0\right\rangle=O_{1}\left(t, s, z^{*}, w^{*}\right)\langle z|\left\langle w\left|\mathcal{U}_{t}\right| 0\right\rangle
$$

and

$$
B(s)=\langle z|\left\langle w\left|\mathcal{U}_{t} a^{\dagger}(s)\right| 0\right\rangle=O_{2}\left(t, s, z^{*}, w^{*}\right)\langle z|\left\langle w\left|\mathcal{U}_{t}\right| 0\right\rangle
$$

where $\mathcal{U}_{t}$ is the unitary operator for the extended system (14): $\left|\psi_{\text {tot }}(t)\right\rangle=\mathcal{U}_{t}\left|\psi_{\text {tot }}(0)\right\rangle$. Clearly, the operators $A(s)$ and $B(s)$ depend on the time $t$, but for our purpose, we regard them, and therefore also the operators $O_{i}\left(t, s, z^{*}, w^{*}\right)$, as functions of $s$. The time $t$ appears as a parameter only. Obviously, the evolution equations for $A(s)$ and $B(s)$ are equivalent to that for $O_{1}\left(t, s, z^{*}, w^{*}\right)$ and $O_{2}\left(t, s, z^{*}, w^{*}\right)$ with respect to $s$, respectively. First, note that the Heisenberg equation of motion for $a(s)$ gives rise to

$$
i \partial_{s} a(s)=\Omega a(s)-\sum_{\lambda} \sqrt{\bar{n}_{\lambda}+1} g_{\lambda} e^{-i \omega_{\lambda} s} d_{\lambda}(s)+\sum_{\lambda} \sqrt{\bar{n}_{\lambda}} g_{\lambda} e^{-i \omega_{\lambda} s} e_{\lambda}^{\dagger}(s)
$$

Therefore, we get

$$
\begin{aligned}
i \partial_{s} A(s)=\Omega A(s) & +\sum_{\lambda} \sqrt{\bar{n}_{\lambda}+1} g_{\lambda} e^{-i \omega_{\lambda} s}\langle z|\left\langle w\left|\mathcal{U}_{t} d_{\lambda}(s)\right| 0\right\rangle \\
& +\sum_{\lambda} \sqrt{\bar{n}_{\lambda}} g_{\lambda} e^{-i \omega_{\lambda} s}\langle z|\left\langle w\left|\mathcal{U}_{t} e_{\lambda}^{\dagger}(s)\right| 0\right\rangle
\end{aligned}
$$

Simply integrating the Heisenberg equation of motion for the environmental annihilation operator $d_{\lambda}(s)$, we get

$$
\langle z|\left\langle w\left|\mathcal{U}_{t} d_{\lambda}(s)\right| 0\right\rangle=-i g_{\lambda}^{*} \sqrt{\bar{n}_{\lambda}+1} \int_{0}^{s} d s^{\prime} e^{i \omega_{\lambda} s^{\prime}}\langle z|\left\langle w\left|\mathcal{U}_{t} a\left(s^{\prime}\right)\right| 0\right\rangle,
$$

where we have used the fact that initially $d_{\lambda}(0)|0\rangle=d_{\lambda}|0\rangle=0$.

Next, we note that the operator $e_{\lambda}^{\dagger}(s)$ in (153) can be dealt with in a similar fashion. In order to find a closed equation, however, we have to carry out an expansion at the final time $t$ rather than the initial value $s=0$. This simplifies the calculations: $\langle z|\left\langle w\left|\mathcal{U}_{t} e_{\lambda}^{\dagger}(t)\right| 0\right\rangle=$ $w_{\lambda}^{*}\langle z|\left\langle w\left|\mathcal{U}_{t}\right| 0\right\rangle$. Thus, we integrate the Heisenberg equation of motion for $e_{\lambda}^{\dagger}(s)$ given the final value at $s=t$ to get

$$
e_{\lambda}^{\dagger}(s)=e_{\lambda}^{\dagger}(t)-i \sqrt{\bar{n}_{\lambda}} g_{\lambda}^{*} \int_{s}^{t} d s^{\prime} e^{-i \omega_{\lambda} s^{\prime}} a\left(s^{\prime}\right),
$$


hence, we get

$$
\langle z|\left\langle w\left|\mathcal{U}_{t} e_{\lambda}^{\dagger}(s)\right| 0\right\rangle=w_{\lambda}^{*}\langle z|\left\langle w\left|\mathcal{U}_{t}\right| 0\right\rangle-i \sqrt{\bar{n}_{\lambda}} g_{\lambda} \int_{s}^{t} d s^{\prime} e^{-i \omega_{\lambda} s^{\prime}}\langle z|\left\langle w\left|\mathcal{U}_{t} a\left(s^{\prime}\right)\right| 0\right\rangle .
$$

Combining the results (53), (54), and (56), we obtain a linear first-order differential equation for $A(s)$ :

$$
\begin{aligned}
& \partial A(s)+\Omega A(s) \\
& -i \int_{0}^{s} d s^{\prime} \alpha_{1}\left(s-s^{\prime}\right) A\left(s^{\prime}\right)-i \int_{s}^{t} d s^{\prime} \alpha_{2}\left(s^{\prime}-s\right) A\left(s^{\prime}\right)=-i w_{s}^{*}\langle z|\left\langle w\left|\mathcal{U}_{t}\right| 0\right\rangle,
\end{aligned}
$$

for $s \in[0, t]$ and a fixed final time $t$. Note that equation (57) has to be solved with the final value $A(s=t)=a\langle z|\left\langle w\left|\mathcal{U}_{t}\right| 0\right\rangle$.

By noting that $A(s)=O_{1}\left(t, s, z^{*}, z^{*}\right)\langle z|\left\langle w\left|\mathcal{U}_{t}\right| 0\right\rangle$ from (50), so with the derived first order differential equation for $A(s)$, we may immediately obtain the evolution equation with respect to $s$ for the desired operator $O_{1}\left(t, s, z^{*}, w^{*}\right)$, we find

$$
\begin{aligned}
i \partial_{s} O_{1}\left(t, s, z^{*}, w^{*}\right)= & \Omega O_{1}\left(t, s, z^{*}, w^{*}\right)-i \int_{0}^{s} d s^{\prime} \alpha_{1}\left(s-s^{\prime}\right) O_{1}\left(t, s^{\prime}, z^{*}, w^{*}\right) \\
& -i \int_{s}^{t} d s^{\prime} \alpha_{2}\left(s^{\prime}-s\right) O_{1}\left(t, s^{\prime}, z^{*}, w^{*}\right)+i w_{s}^{*}
\end{aligned}
$$

with the final value

$$
O_{1}\left(t, s=t, z^{*}, w^{*}\right)=a
$$

Similarly, we get the evolution equation for $\mathrm{O}_{2}\left(t, s, z^{*}, w^{*}\right)$ :

$$
\begin{aligned}
i \partial_{s} O_{2}\left(t, s, z^{*}, w^{*}\right)= & -\Omega O_{2}\left(t, s, z^{*}, w^{*}\right)+i \int_{0}^{s} d s^{\prime} \alpha_{2}\left(s-s^{\prime}\right) O_{2}\left(t, s^{\prime}, z^{*}, w^{*}\right) \\
& +i \int_{s}^{t} d s^{\prime} \alpha_{1}\left(s^{\prime}-s\right) O_{2}\left(t, s^{\prime}, z^{*}, w^{*}\right)-i z_{s}^{*}
\end{aligned}
$$

with the final value

$$
O_{2}\left(t, s=t, z^{*}, w^{*}\right)=a^{\dagger}
$$

Remarkably, we see that the coupled nonlinear equations (28) and (29) have become a set of uncoupled linear equations (58) and (60). We emphasize that equations (58) and (60), with the final values (59) and (61) respectively, are all that is required for the derivation of the master equation for the damped harmonic oscillator. 
The equations (58) and (60) in terms of $f_{1}(t, s), f_{2}(t, s)$ and $h_{1}\left(t, s, s^{\prime}\right), h_{2}\left(t, s, s^{\prime}\right)$ can be written as a set of uncoupled linear equations for $s \in[0, t]$, with $t$ fixed,

$$
\begin{gathered}
\partial_{s} f_{1}(t, s)+i \Omega f_{1}(t, s)+\int_{0}^{s} d s^{\prime} \alpha_{1}\left(s-s^{\prime}\right) f_{1}\left(t, s^{\prime}\right)+\int_{s}^{t} d s^{\prime} \alpha_{2}\left(s^{\prime}-s\right) f_{1}\left(t, s^{\prime}\right)=0 \\
\partial_{s} f_{2}(t, s)-i \Omega f_{2}(t, s)-\int_{0}^{s} d s^{\prime} \alpha_{2}\left(s-s^{\prime}\right) f_{2}\left(t, s^{\prime}\right)-\int_{s}^{t} d s^{\prime} \alpha_{1}\left(s^{\prime}-s\right) f_{2}\left(t, s^{\prime}\right)=0 \\
\partial_{s} j_{1}\left(t, s, s^{\prime}\right)+i \Omega j_{1}\left(t, s, s^{\prime}\right)+\int_{0}^{s} d s^{\prime \prime} \alpha_{1}\left(s-s^{\prime \prime}\right) j_{1}\left(t, s^{\prime \prime}, s^{\prime}\right) \\
+\int_{s}^{t} d s^{\prime \prime} \alpha_{2}\left(s^{\prime \prime}-s\right) j_{1}\left(t, s^{\prime \prime}, s^{\prime}\right)=\delta\left(s-s^{\prime}\right) \\
\partial_{s} j_{2}\left(t, s, s^{\prime}\right)-i \Omega j_{2}\left(t, s, s^{\prime}\right)-\int_{0}^{s} d s^{\prime \prime} \alpha_{2}\left(s-s^{\prime \prime}\right) j_{2}\left(t, s^{\prime \prime}, s^{\prime}\right) \\
-\int_{s}^{t} d s^{\prime \prime} \alpha_{1}\left(s^{\prime \prime}-s\right) j_{2}\left(t, s^{\prime \prime}, s^{\prime}\right)=-\delta\left(s-s^{\prime}\right)
\end{gathered}
$$

with the final values

$$
\begin{aligned}
& f_{1}(t, s=t)=1, \quad f_{2}(t, s=t)=1 \\
& j_{1}(t, t, s)=0, \quad j_{2}(t, t, s)=0, \quad \text { for all } s .
\end{aligned}
$$

\section{Master equation for the damped harmonic oscillator}

Now we are going to derive the exact master equation for the damped harmonic oscillator. We will see that the evolution equations of $O_{i}$ are crucial for deriving the corresponding nonMarkovian master equation from the stochastic schrodinger equation (40). From (30), we get

$$
\begin{aligned}
\partial_{t} \rho_{t}= & -i \Omega\left[a^{\dagger} a, \rho_{t}\right]+\left[a, \mathcal{M}\left\{P_{t} \bar{O}_{1}^{\dagger}\left(t, z^{*}, w^{*}\right)\right\}\right]-\left[a^{\dagger}, \mathcal{M}\left\{\bar{O}_{1}\left(t, z^{*}, w^{*}\right) P_{t}\right\}\right] \\
& +\left[a^{\dagger}, \mathcal{M}\left\{P_{t} \bar{O}_{2}^{\dagger}\left(t, z^{*}, w^{*}\right)\right\}\right]-\left[a, \mathcal{M}\left\{\bar{O}_{2}\left(t, z^{*}, w^{*}\right) P_{t}\right\}\right]
\end{aligned}
$$

For the equation (68) to be a closed, convolutionless master equation, we need the explicit expressions for the ensemble means:

$$
R_{1}(t, s) \equiv \mathcal{M}\left[O_{1}\left(t, s, z^{*}, w^{*}\right) P_{t}\right], \quad R_{2}(t, s) \equiv \mathcal{M}\left[O_{2}\left(t, s, z^{*}, w^{*}\right) P_{t}\right]
$$

For this purpose, we take the mean $\mathcal{M}\left[\cdots P_{t}\right]$ of equation (58), and then we get

$$
\begin{aligned}
& \partial_{s} R_{1}(t, s)+i \Omega R_{1}(t, s) \\
& +i \int_{0}^{s} d s^{\prime} \alpha_{1}\left(s-s^{\prime}\right) R_{1}\left(t, s^{\prime}\right)-i \int_{s}^{t} d s^{\prime} \alpha_{2}\left(s^{\prime}-s\right) R_{1}\left(t, s^{\prime}\right)=-i \mathcal{M}\left[w_{s}^{*} P_{t}\right] .
\end{aligned}
$$


Thus, we have to find an expression for $\mathcal{M}\left[z_{\mathrm{c}}^{*} P_{t}\right]$. By using Novikov's theorem [33], it is possible to relate it to the $O$-operator again [20]:

$$
\mathcal{M}\left[w_{s}^{*} P_{t}\right]=\int_{0}^{t} d s^{\prime} \alpha_{2}^{*}\left(s-s^{\prime}\right) \mathcal{M}\left[P_{t} O_{2}^{\dagger}\left(t, s^{\prime}, z^{*}, w^{*}\right)\right]=\int_{0}^{t} d s^{\prime} \alpha_{2}^{*}\left(s-s^{\prime}\right) R_{2}^{\dagger}\left(t, s^{\prime}\right) .
$$

Thus, we find the equations for the operator $R_{1}(t, s)$, as function of $s$, to be

$$
\begin{aligned}
& \partial_{s} R_{1}(t, s)+i \Omega R_{1}(t, s) \\
& +\int_{0}^{s} d s^{\prime} \alpha_{1}\left(s-s^{\prime}\right) R_{1}\left(t, s^{\prime}\right)+\int_{s}^{t} d s^{\prime} \alpha_{2}\left(s^{\prime}-s\right) R_{1}\left(t, s^{\prime}\right)=\int_{0}^{t} d s^{\prime} \alpha_{2}^{*}\left(t-s^{\prime}\right) R_{2}^{\dagger}\left(t, s^{\prime}\right) .
\end{aligned}
$$

and similarly, for the operator $R_{2}(t, s)$, we have

$$
\begin{aligned}
\partial_{s} R_{2}(t, s) & -i \Omega R_{2}(t, s)-\int_{0}^{s} d s^{\prime} \alpha_{2}\left(s-s^{\prime}\right) R_{2}\left(t, s^{\prime}\right) \\
& -\int_{s}^{t} d s^{\prime} \alpha_{1}\left(s^{\prime}-s\right) R_{2}\left(t, s^{\prime}\right)=-\int_{0}^{t} d s^{\prime} \alpha_{1}^{*}\left(s-s^{\prime}\right) R_{1}^{\dagger}\left(t, s^{\prime}\right) .
\end{aligned}
$$

Note that the equations (72) and (173) have to be solved with "final values"

$$
\left.R_{1}(t, s)\right|_{s=t}=a \rho_{t},\left.\quad R_{2}(t, s)\right|_{s=t}=a^{\dagger} \rho_{t},
$$

as one can easily see from (59) and (61). Note that equations (172) and (73) are a set of coupled linear equations that are expected from (41) and (42).

By observing (41) and (42), we find that the solutions of the crucial equations (72) and (73) with the final values (744) must take the following form:

$$
\begin{aligned}
& R_{1}(t, s)=F(t, s) a \rho_{t}+G(t, s) \rho_{t} a \\
& R_{2}(t, s)=H(t, s) a^{\dagger} \rho_{t}+I(t, s) \rho_{t} a^{\dagger}
\end{aligned}
$$

where $F(t, s), G(t, s), H(t, s)$, and $I(t, s)$ are complex functions with the appropriate final values at $s=t$ :

$$
\begin{aligned}
& F(t, s=t)=1, \quad G(t, s=t)=0 \\
& H(t, s=t)=1, \quad I(t, s=t)=0
\end{aligned}
$$

Once we have $\left.R_{i}(t, s)=\mathcal{M}\left[O_{i}\left(t, s, z^{*}, w^{*}\right) P_{t}\right](i=1,2)\right)$ in the form of (75) and (176), it is straightforward to write down the master equation. Simply inserting result (75) and (76) into the general form of the non-Markovian QSD-master equation (68), we get

$$
\partial_{t} \rho_{t}=-i \Omega\left[a^{\dagger} a, \rho_{t}\right]+a(t)\left[a, \rho a^{\dagger}\right]+b(t)\left[a, a^{\dagger} \rho\right]+c(t)\left[a^{\dagger}, a \rho\right]+d(t)\left[a^{\dagger}, \rho a\right],
$$


with

$$
\begin{aligned}
a(t) & =\int_{0}^{t} d s\left(\alpha_{1}^{*}(t-s) F^{*}(t, s)-\alpha_{2}(t-s) I(t, s)\right), \\
b(t) & =\int_{0}^{t} d s\left(\alpha_{1}^{*}(t-s) G^{*}(t, s)-\alpha_{2}(t-s) H(t, s)\right), \\
c(t) & =-a^{*}(t), \\
d(t) & =-b^{*}(t) .
\end{aligned}
$$

The equation (79) is valid for arbitrary temperature, so it is expected to provide a good description for the low temperature regimes where the Markov approximation is doomed to fail. Clearly, the master equation (79) derived in this way automatically preserves the positivity, Hermiticity and trace. Moreover, it can be easily shown that the above master

equation is of the Lindblad form, but the coefficients are time-dependent, so it depicts the non-Markovian dynamics.

\section{Determination of the coefficients of master equation}

In what follows, we show that the coefficients $a(t), b(t), c(t)$ and $d(t)$ can be expressed in terms of solutions of some basic equations. Suppose that $u(t, s)$ is the solution to the following homogeneous equation:

$$
\partial_{s} u(t, s)-i \Omega u(t, s)+\int_{s}^{t} d s^{\prime} \beta\left(s, s^{\prime}\right) u\left(t, s^{\prime}\right)=0,
$$

with the final value $u(t, s=t)=1$. Here the kernel function $\beta\left(s, s^{\prime}\right)$ is defined as

$$
\beta\left(s, s^{\prime}\right)=\alpha_{2}\left(s-s^{\prime}\right)-\alpha_{1}\left(s^{\prime}-s\right) .
$$

Then from (72) and (73) it can be shown that $F(t, s), G(t, s), H(t, s)$ and $I(t, s)$ satisfy the following inhomogeneous equation,

$$
\begin{gathered}
\partial_{s} F(t, s)+i \Omega F(t, s)-\int_{0}^{s} d s^{\prime} \beta\left(s^{\prime}, s\right) F\left(t, s^{\prime}\right)=-X(t, s), \\
\partial_{s} G(t, s)+i \Omega G(t, s)-\int_{0}^{s} d s^{\prime} \beta\left(s^{\prime}, s\right) G\left(t, s^{\prime}\right)=X(t, s), \\
\partial_{s} H(t, s)-i \Omega H(t, s)-\int_{0}^{s} d s^{\prime} \beta\left(s, s^{\prime}\right) H\left(t, s^{\prime}\right)=Y(t, s), \\
\partial_{s} I(t, s)-i \Omega I(t, s)-\int_{0}^{s} d s^{\prime} \beta\left(s, s^{\prime}\right) I\left(t, s^{\prime}\right)=-Y(t, s),
\end{gathered}
$$


where the functions $X(t, s)$ and $Y(t, s)$ are given by

$$
\begin{aligned}
& X(t, s)=\int_{0}^{t} d s^{\prime} \alpha_{2}\left(s^{\prime}-s\right) u^{*}\left(t, s^{\prime}\right), \\
& Y(t, s)=\int_{0}^{t} d s^{\prime} \alpha_{1}\left(s^{\prime}-s\right) u\left(t, s^{\prime}\right) .
\end{aligned}
$$

Hence it is easy to see that the coefficients of the master equation (79) can be expressed in terms of the solutions of those basic equations (84) and (866)-(89).

\section{CONCLUSIONS}

The non-Markovian quantum trajectories offer a brand new method for exploring a quantum system coupled to a non-Markovian environment. Such a situation appears in various physical problems, such as materials with photonic band gaps or output of atom lasers. It is known that master equations and quantum trajectories in Markov regimes are of fundamental importance for the description of open system dynamics. Moreover, they are often complementary to each other to provide a full picture of the underlying physics. In this paper we show that in the case of general finite temperature heat bath this fruitful interrelation between the master equations and the quantum trajectories can also be established in the non-Markovian regimes. In particular, we show that by averaging the stochastic Schrödinger equation the exact non-Markovian master equation of the damped harmonic oscillator at finite temperature can be obtained. The master equation derived in this way takes the convolutionless form, so the non-Markovian property is encoded in the time-dependent coefficients.

In the Markov regimes, the derivation of a master equation poses no special difficulties. However, it is a rather difficult task to derive a convolutionless exact master equation in the non-Markovian regimes. As shown in this paper, non-Markovian quantum trajectories provide a very useful tool in handling exact or approximate non-Markovian master equations. We believe that the techniques employed in this paper may find broader applications to other quantum open systems, leading to the establishment of a general master equation for the system interacting with a bosonic heat bath. This will be the topic of future investigations. 


\section{Acknowledgments}

I thank Lajos Diósi, Ian Percival and Walter Strunz for valuable discussions and I particularly thank Joseph Eberly for reading the manuscript and for his critical comments. Grants from the NEC Research Institute (US) and Leverhulme Foundation (UK) provided financial support.

[1] C. Gardiner and P. Zoller, Quantum Noise, second edition, (Springer, Berlin) (2000).

[2] H. Carmichael, Statistical Methods in Quantum Optics I, (Springer, Berlin) (2000).

[3] C. Cohen-Tannoudji, J. Dupont-Roc, G. Grynberg, Atom and Photon Interactions: Basic Processes and Applications, (John Wiley \& Sons, Inc. New York ) (1998).

[4] G. Lindblad, Comm. Math. Phys. 48, 119 (1976).

[5] V. Gorini, A. Kossakowski, and E. C. G. Sudarshan, J. Math. Phys. 17, 821 (1976).

[6] H. Carmichael, An Open System Approach to Quantum Optics, (Springer, Berlin) (1993).

[7] J. Dalibard, Y. Castin, and K. Mølmer, Phys. Rev. Lett. 68580 (1992).

[8] N. Gisin and I. C. Percival, J. Phys. A 25, 5677 (1992); 26, 2233 (1993).

[9] C. Gardiner, A. Parkins, and P. Zoller, Phys. Rev. A 46, 4363 (1992).

[10] H. M. Wiseman and G. J. Milburn, Phys. Rev. A 47, 1652 (1993).

[11] M. Plenio and P. L. Knight, Rev. Mod. Phys. 70, 101 (1998).

[12] I. C. Percival, Quantum State Diffusion, (Cambridge, England) (1998).

[13] S. John and T. Quang, Phys. Rev. Lett. 74, 3419; B. M. Garraway, Phys. Rev. A 55, 4636 (1997); M. Nikolopoulos and P. Lambroupoulos, Phys. Rev. A 61, 053812 (2000).

[14] J. J. Hope, Phys. Rev. A 55, R2531 (1997); H.-P. Breuer, D. Faller, B. Kappeler, and F. Petruccione, Phys. Rev. A 60, 3188 (1999).

[15] S. Nakajima, Prog. Theor. Phys. 20, 948 (1958).

[16] R. Zwanzig, J. Chem. Phys. 33, 1338 (1960).

[17] L. Diósi and W. T. Strunz, Phys. Lett. A 235, 569 (1997).

[18] L. Diósi, N. Gisin and W. T. Strunz, Phys. Rev. A 58, 1699 (1998).

[19] W. T. Strunz, L. Diósi, and N. Gisin, Phys. Rev. Lett. 82, 1801 (1999).

[20] T. Yu, L. Diósi, N. Gisin and W. T. Strunz, Phys. Rev. A 60, 91 (1999). 
[21] W. T. Strunz, L. Diósi, N. Gisin and T. Yu, Phys. Rev. Lett. 83, 4909 (1999).

[22] H. Breuer, B. Kappler, and F. Petruccione, Phys. Rev. A 59, 1633 (1999).

[23] M. Jack, M. Collet, and D. Walls. Phys. Rev. A 59, 2308 (1999).

[24] J. Cresser, Laser Phys. 10, 1 (2000).

[25] A. A. Budini, Phys. Rev. A 63, 012106 (2000).

[26] J. Gambetta and H. M. Wiseman, Phys. Rev. A 66, 012108 (2002); 66, 052105 (2002); 68, $062104(2003)$.

[27] A. Bassi, Phys. Rev. A 67, 062101 (2003).

[28] T. Yu, L. Diósi, N. Gisin and W. T. Strunz, Phys. Lett. A 265, 331 (2000).

[29] W. T. Strunz and T. Yu, Preprint, arXiv: quant-ph/0312103 (2003), Physical Review A (to appear).

[30] For the quantum Brownian motion model, a collection of references are as follows: R. Feynman and F. L. Vernon, Ann. Phys. (N.Y.) 24, 118 (1963); A. O. Caldeira and A. J. Leggett, Physica A 121, 587 (1983); H. Grabert, P. Schramm, and G.-L. Ingold, Phys. Rep. 168, 115 (1988); F. Haake and R. Reibold, Phys. Rev. A 32, 2462 (1985); W. G. Unruh and W. Zurek, Phys. Rev. D 40, 1071 (1989); B. L. Hu, J. P. Paz and Y. Zhang, Phys. Rev. D 45, 2843 (1992); Phys. Rev. D 47, 1576 (1993); J. J. Halliwell and T. Yu, Phys. Rev. D 53, 2012 (1996); G. W. Ford and R. F. O'Connell, Phys. Rev. D 64, 105020 (2001).

[31] G. W. Semenoff and H. Umezawa, Nucl. Phys. B 220, 196 (1983).

[32] T. Yu and J. H. Eberly, Phys. Rev. B 66, 193306 (2002).

[33] A. Novikov, Sov. Phys. JETP 20, 1290 (1965).

[34] More accurately, the numerical simulations need the nonlinear version of the non-Markovian QSD equation which can be read off directly from (40) 